\title{
A Rare Case of Intraspinal Psammomatous Melanotic Schwannoma: A Case Report
}

\author{
Naoki Takatori, Akihiko Hiyama, Daisuke Sakai, Hiroyuki Katoh, Masato Sato and Masahiko Watanabe \\ Department of Orthopaedic Surgery, Surgical Science, Tokai University School of Medicine, Isehara, Japan
}

\begin{abstract}
Keywords:
psammomatous melanotic schwannoma, melanotic schwannoma, intraspinal tumor, cauda equina

Spine Surg Relat Res 2020; 4(1): 91-94 dx.doi.org/10.22603/ssrr.2019-0034
\end{abstract}

Psammomatous melanotic schwannoma (PMS) is a rare tumor originating in the peripheral nerve sheath. The standard treatment for PMS is total resection. Herein, we report a case of PMS.

A 39-year-old male was referred to our hospital after suffering from low back pain and numbness of the left leg. Radiography and CT revealed scalloping and bone erosion of the L4 vertebral body with mineralization within the tumor (Fig. 1). MRI demonstrated a dumbbell tumor at the L4 level with high T1- and low T2-weighted signal intensities and homogenous contrast enhancement after gadolinium infusion (Fig. 2). Surgery was planned to resect the lumbar dumbbell tumor (Eden type 2). A L3-L4 laminectomy revealed a heavily pigmented, almost entirely dark black tumor generating from the right L4 nerve root. When the dura was incised, extensive cauda equina invasion was observed (Fig. 3A), rendering complete resection impossible. Therefore, it was decided to resect as much of the tumor as possible while preserving spinal function.

Staining of the tumor sections revealed nuclear atypia and mitosis. We performed antimelanoma antibody (HMB-45) staining to reveal the presence of melanin. HMB-45 staining is usually strongly positive in primary melanocytic lesions of the central nervous system. In addition, fibrous components of the tumor sections stained positive for laminin, which is a characteristic for schwannomas. Based on these morphological and immunohistochemical characteristics, the diagnosis of PMS was made (Fig. 3B, C). Surgery improved the patient's symptoms, but adjuvant radiotherapy was performed to address the concerns of recurrence from the residual tumor. Nine months after surgery, the patient exhibited progressive lower limb weakness and paresthesia. Radiogra- phy revealed expansion of the L4 lumbar vertebra scalloping and multiple lung metastases (Fig. 4). The patient died 22 months after surgery. Autopsy revealed widely disseminated metastatic tumors involving the lungs, spinal cord, bilateral chest wall, and stomach. The direct cause of death was thought to be metastasis or the dissemination of PMS.

To date, $<200$ cases have been reported, and the overall prevalence of melanotic schwannoma (MS) in primary peripheral nerve sheath tumors is $\left\langle 1 \%^{1)}\right.$. The average age at diagnosis is approximately $30-40$ years. Furthermore, the development of these tumors is not associated with $\operatorname{sex}^{2,3)}$. MS is generally considered as benign ${ }^{3}$ and malignant in approximately $10 \%-15 \%{ }^{4}$. On MRI, conventional schwannomas display low T1-weighted and high T2-weighted signal intensities. Conversely, MS exhibits high T1-weighted and low T2weighted signal intensities because of the paramagnetic free radicals in melanin ${ }^{5)}$. This pattern is rarely found and therefore is an important point leading to the suspicion of MS. Approximately $40 \%-50 \%$ of MS are psammomatous ${ }^{6,7)}$; approximately half of these cases are idiopathic, whereas the rest are associated with Carney complex ${ }^{7,8)}$. Carney complex is an autosomal dominant syndrome characterized by spotty skin pigmentation, endocrine tumors, and myxomas of the heart, skin, and breast ${ }^{6,7}$. Physical examination, laboratory data, and radiographic findings of our patient excluded Carney complex.

Although the standard treatment for PMS is total resection, the rate of recurrence is higher than that in non-PMS even when complete resection is achieved ${ }^{8)}$, thus raising the question whether to perform postsurgical radiotherapy and/or chemotherapy. Some studies have suggested that radiotherapy should be administered to patients in whom only partial

Corresponding author: Naoki Takatori, n.tori001@tsc.u-tokai.ac.jp

Received: May 10, 2019, Accepted: June 17, 2019, Advance Publication: July 10, 2019

Copyright (C) 2020 The Japanese Society for Spine Surgery and Related Research 

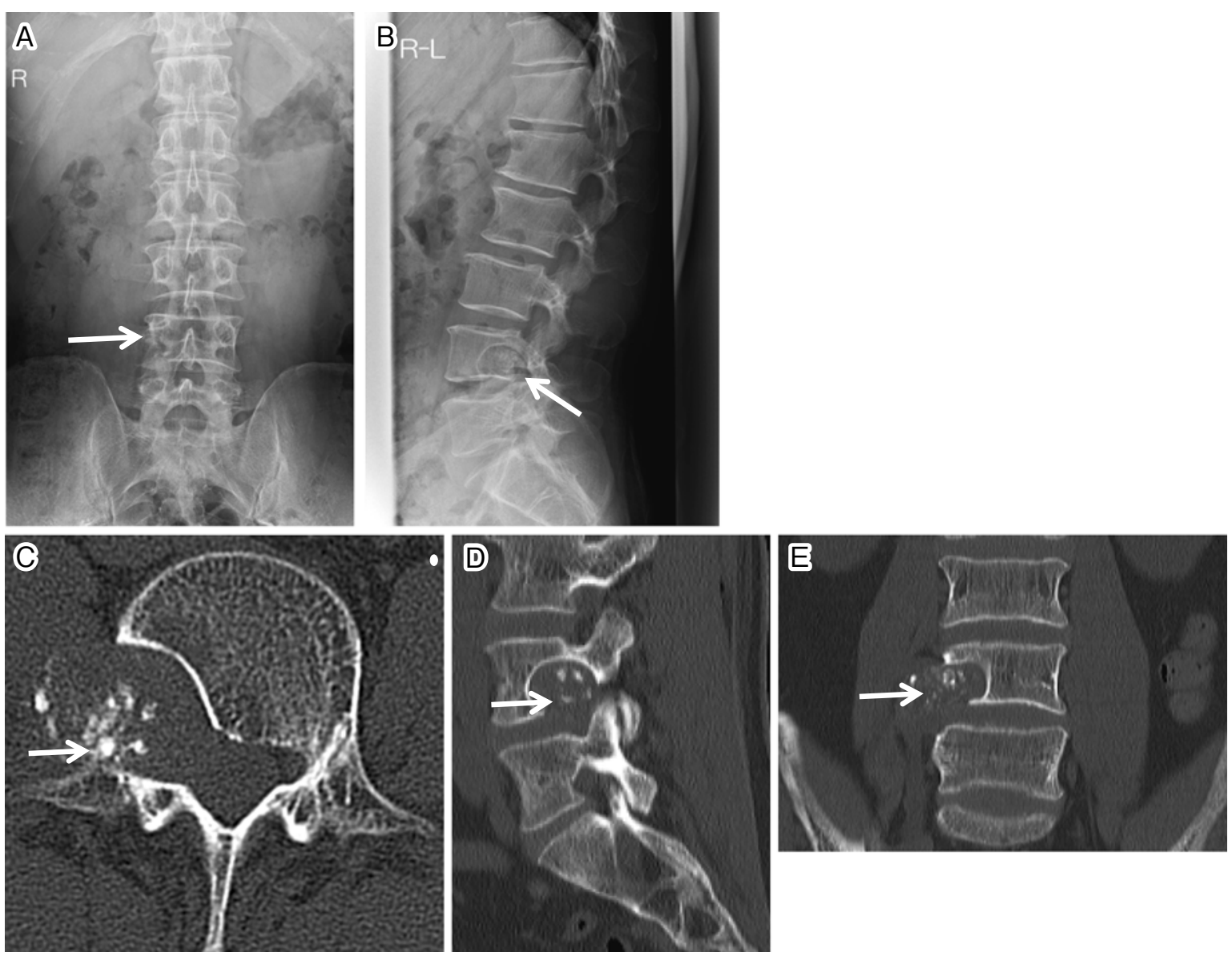

Figure 1. (A) Scalloping of the L4 vertebral body and the psammoma body observed in the tumor (arrow) by anterioposterior, (B) lateral view. (C) Computed tomography (CT) axial view reveals scalloping of the L4 vertebra and psammoma bodies in the tumor (arrow), (D) sagittal view, (E) coronal view.
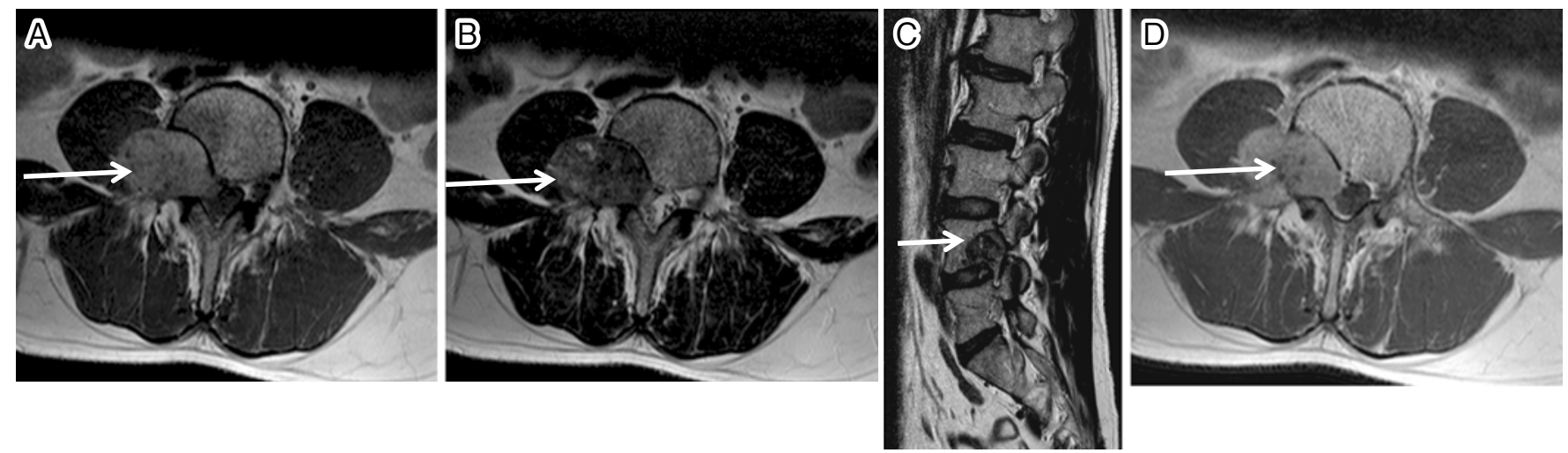

Figure 2. Magnetic resonance imaging (MRI) reveals a tumor with high T1-weighted and low T2-weighted signal intensities and homogenous gadolinium enhancement. (A) T1-weighted axial image, (B) T2-weighted axial image, (C) T2-weighted sagittal image, and (D) gadolinium enhanced T1-weighted axial image.

resection is possible to decrease the likelihood of metastasis $^{9)}$. Radiotherapy was used as a secondary treatment in our case because only partial resection was achieved, but progressive tumor growth and metastasis were observed. Complete resection of PMS tumors in the cauda equina is often difficult, with the residual tumor leading to acute progression of the disease. Therefore, early diagnosis is paramount for successful treatment. Partially resected tumors may require additional radiotherapy and/or chemotherapy, but their efficacy is limited.

Conflicts of Interest: The authors declare that there are no relevant conflicts of interest. 

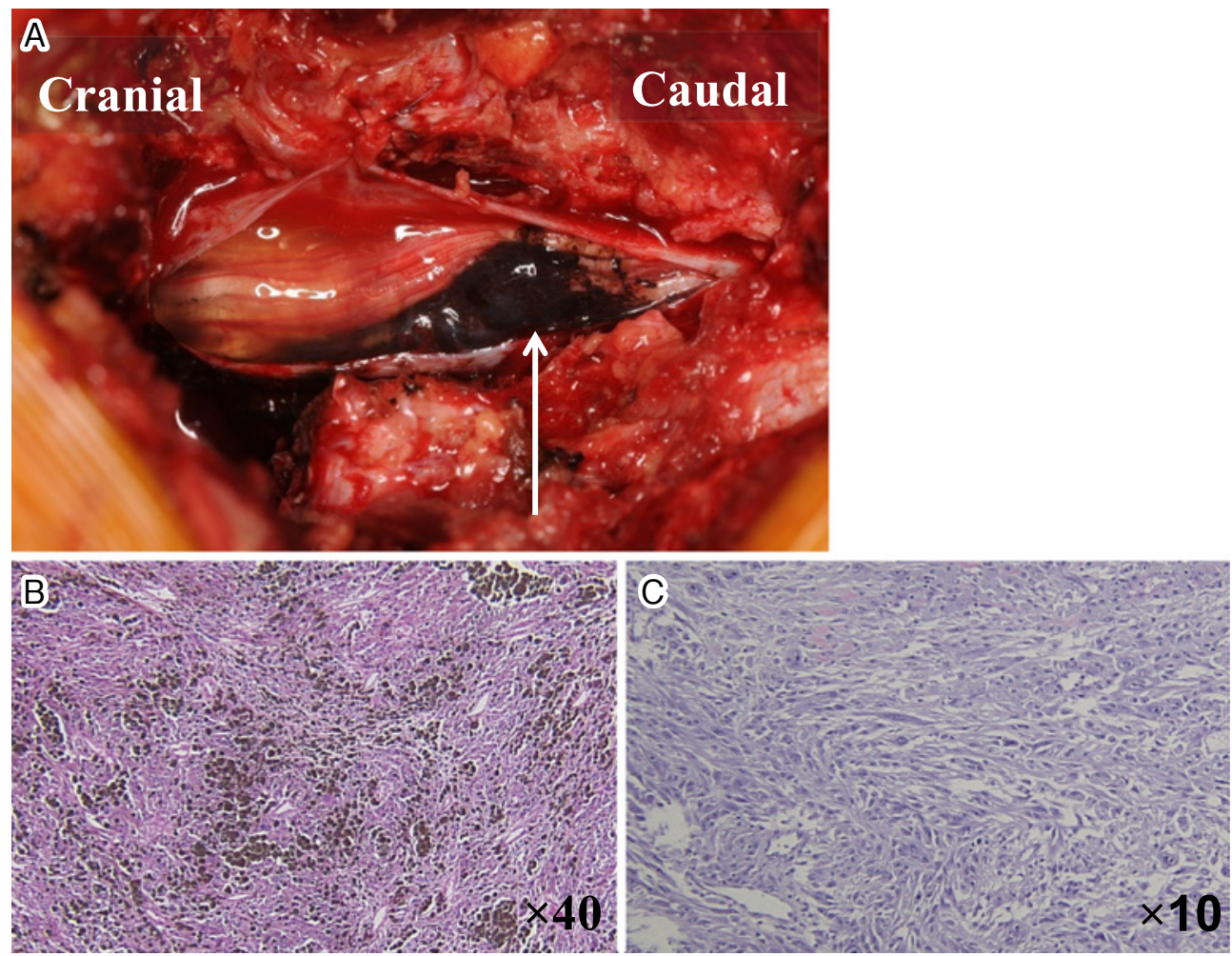

Figure 3. (A) Intraoperative photograph showing the black tumor within the dura mater. Tumor pathology. (B) Hematoxylin and eosin staining, 40× magnification and (C) de-melanin staining, $100 \times$ magnification.
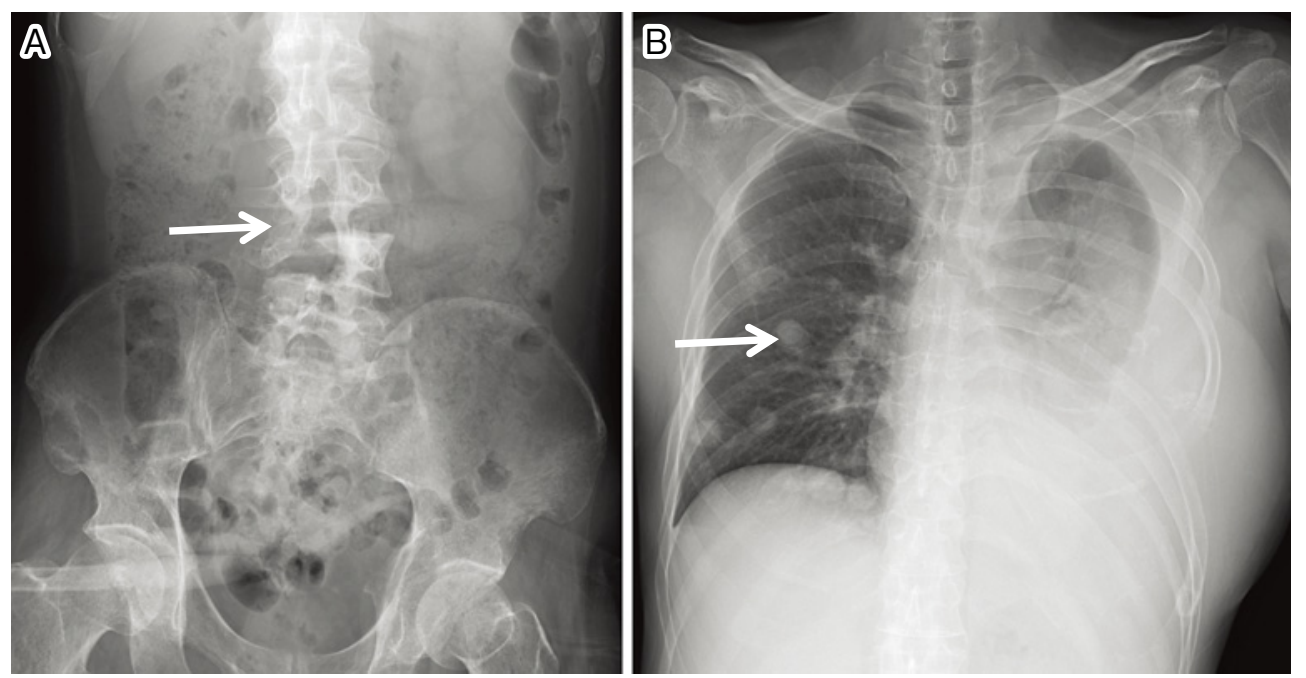

Figure 4. X-ray of the chest and spine showing (A) expansion of scalloping and (B) multiple metastasis within the lung.

Author Contributions: Naoki Takatori and Akihiko Hiyama drafted this case report. Daisuke Sakai, Hiroyuki Katoh, Masato Sato, and Masahiko Watanabe revised the drafted paper. All authors gave final approval of the version to be published.

Informed Consent: Informed consent was obtained from all participants in this study.

\section{References}

1. Khoo M, Pressney I, Hargunani R, et al. Melanotic schwannoma: an 11-year case series. Skeletal Radiol. 2016;45(1):29-34.

2. Er U, Kazanci A, Eyriparmak T, et al. Melanotic schwannoma. J Clin Neurosci. 2007;14(7):676-8.

3. Vallat-Decouvelaere AV, Wassef M, Lot G, et al. Spinal melanotic schwannoma: a tumour with poor prognosis. Histopathology. 1999;35(6):558-66.

4. Xing C, Jiagang L, Jun Le, et al. Invasive intramedullary 
melanotic schwannoma: case report and review of the literature. Eur Spine J. 2018;27(suppl 3):S303-8.

5. Hoover JM, Bledsoe JM, Giannini C, et al. Intramedullary melanotic schwannoma. Rare Tumors. 2012;4(1):e3.

6. Carney JA. Psammomatous melanotic schwannoma: A distinctive, heritable tumor with special associations, including cardiac myxoma and the Cushing syndrome. Am J Surg Pathol. 1990;14 (3):206-22.

7. Stratakis CA, Raygada M. GeneReviews ${ }^{\circledR}$ [Internet]. Seattle (WA): University of Washington; 1993-2019 [updated 2015 Jan 29]. Carney complex. Available from: https://www.ncbi.nlm.nih.gov/books/
NBK1286/

8. Shabani S, Fiore SM, Seidman R, et al. Intraspinal psammomatous melanotic schwannoma not associated with Carney complex: case report. J Neurosurg Spine. 2015;23(2):233-8.

9. Santaguida C, Sabbagh AJ, Guidot MC, et al. Aggressive intramedullary melanotic schwannoma. Neurosurgery. 2004;55(6):1430-4.

Spine Surgery and Related Research is an Open Access journal distributed under the Creative Commons Attribution-NonCommercial-NoDerivatives 4.0 International License. To view the details of this license, please visit (https://creativeco mmons.org/licenses/by-nc-nd/4.0/). 